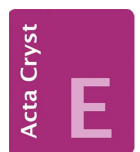

CRYSTALLOGRAPHIC COMMUNICATIONS

ISSN 2056-9890
Received 16 January 2019

Accepted 7 February 2019

Edited by A. V. Yatsenko, Moscow State University, Russia

Keywords: crystal structure; Schiff bases; Hirshfeld surface; hydrogen bonds; stacking interactions.

CCDC reference: 1886956

Supporting information: this article has supporting information at journals.iucr.org/e

\section{Crystal structure and Hirshfeld surface analysis of a Schiff base: (Z)-6-[(5-chloro-2-methoxyanilino)- methylidene]-2-hydroxycyclohexa-2,4-dien-1-one}

\author{
Sibel Demir Kanmazalp, ${ }^{\text {a* }}$ Onur Erman Dogana, ${ }^{\mathrm{b}}$ Volkan Tașdemir, ${ }^{\mathrm{c}}$ Necmi Dege, ${ }^{\mathrm{d} *}$ \\ Erbil Agarar ${ }^{b}$ and Igor O. Fritsky ${ }^{\mathbf{e}_{*}}$
}

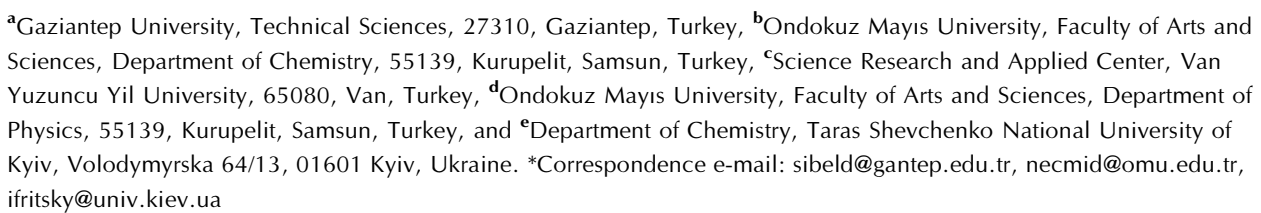

The title compound, $\mathrm{C}_{14} \mathrm{H}_{12} \mathrm{ClNO}_{3}$, is a Schiff base that exists in the ketoenamine tautomeric form and adopts a $Z$ configuration. In the crystal, the dihedral angle between the planes of the benzene rings is $5.34(15)^{\circ}$. The roughly planar geometry of the molecule is stabilized by a strong intramolecular N$\mathrm{H} \cdots \mathrm{O}$ hydrogen bond. In the crystal, pairs of centrosymmetrically related molecules are linked by $\mathrm{O}-\mathrm{H} \cdots \mathrm{O}$ hydrogen bonds, forming $R_{2}^{2}(10)$ rings. Besides this, the molecules form stacks along the [001] direction with $\mathrm{C}-\mathrm{H} \cdots \pi$ and $\mathrm{C}-\mathrm{H} \cdots \mathrm{Cl}$ contacts between the stacks. The intermolecular interactions in the crystal were analysed using Hirshfeld surfaces. The most significant contribution to the crystal packing is from $\mathrm{H} \cdots \mathrm{H}$ contacts $(30.8 \%)$.

\section{Chemical context}

Schiff bases are widely used as ligands in coordination chemistry (Calligaris \& Randaccio, 1987) and they are also of interest in various fields because of their diverse biological activity (Lozier et al., 1975; Costamagna et al., 1992). Some Schiff bases derived from salicylaldehyde have attracted the interest of chemists and physicists because they show thermochromism and photochromism in the solid state (Cohen et al., 1964; Hadjoudis et al., 1987). The origin of their photo- and thermochromism is related to the reversible intramolecular proton transfer associated with a change in the electronic structure (Hadjoudis et al., 1987). The $o$-hydroxy Schiff bases obtained by the condensation of $o$-hydroxyaldehydes with aniline have been extensively examined in this context. Such compounds can exist in two tautomeric forms, viz. keto-enamine $(\mathrm{N}-\mathrm{H} \cdots \mathrm{O})$ and phenol-imine $(\mathrm{N} \cdots \mathrm{H}-$ O) (Stewart \& Lingafelter, 1959; Petek et al., 2010). We report herein the synthesis and the crystal and molecular structures of the title compound, as well as an analysis of its Hirshfeld surfaces.

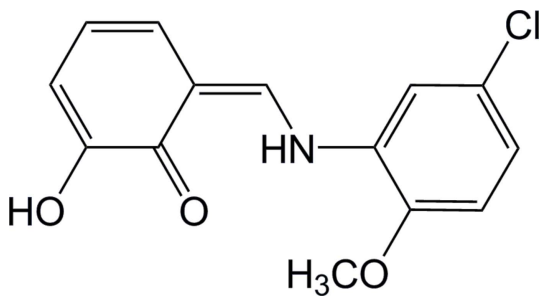


Table 1

Selected bond lengths $(\AA)$.

\begin{tabular}{llll}
\hline $\mathrm{O} 2-\mathrm{C} 9$ & $1.292(4)$ & $\mathrm{C} 8-\mathrm{C} 13$ & $1.410(5)$ \\
$\mathrm{O} 3-\mathrm{C} 10$ & $1.358(4)$ & $\mathrm{C} 9-\mathrm{C} 10$ & $1.426(4)$ \\
$\mathrm{N} 1-\mathrm{C} 6$ & $1.413(4)$ & $\mathrm{C} 10-\mathrm{C} 11$ & $1.359(5)$ \\
$\mathrm{N} 1-\mathrm{C} 7$ & $1.302(4)$ & $\mathrm{C} 11-\mathrm{C} 12$ & $1.402(5)$ \\
$\mathrm{C} 7-\mathrm{C} 8$ & $1.408(4)$ & $\mathrm{C} 12-\mathrm{C} 13$ & $1.349(4)$ \\
$\mathrm{C} 8-\mathrm{C} 9$ & $1.429(4)$ & & \\
\hline
\end{tabular}

\section{Structural commentary}

As shown in Fig. 1., the asymmetric unit of the title compound contains only one molecule, which adopts the keto-enamine tautomeric form: the $\mathrm{H}$ atom is located at $\mathrm{N} 1$, and the lengths of the $\mathrm{N} 1-\mathrm{C} 7$ and $\mathrm{C} 8-\mathrm{C} 9$ bonds indicate their single-bond character, whereas the $\mathrm{O} 2-\mathrm{C} 9$ and $\mathrm{C} 7-\mathrm{C} 8$ bonds are double (Table 1). Overall, the bond lengths in the title structure compare well with those of other keto-enamine tautomers known from the literature (see the Database survey section). The whole molecule is almost planar, with a dihedral angle of $5.34(15)^{\circ}$ between the benzene ring planes. The methoxy $\mathrm{C} 14$ atom deviates from the plane of the $\mathrm{C} 1-\mathrm{C} 6$ benzene ring by 0.038 (4) $\AA$. The torsion angles $\mathrm{C} 1-\mathrm{C} 6-\mathrm{N} 1-\mathrm{C} 7$ and $\mathrm{N} 1-$ $\mathrm{C} 7-\mathrm{C} 8-\mathrm{C} 9$ are $5.8(5)$ and $-0.6(5)^{\circ}$, respectively. The planar molecular conformation is stabilized by the intramolecular $\mathrm{N} 1-\mathrm{H} 2 \cdots \mathrm{O} 2$ hydrogen bond (Table 2).

\section{Supramolecular features}

In the crystal, the molecules are connected via $\mathrm{O}-\mathrm{H} \cdots \mathrm{O}$ hydrogen bonds into centrosymmetric pairs with an $R_{2}^{2}(10)$ graph-set motif (Table 2, Fig. 2). Molecules related by a [001] translation form stacks with an interplanar distance of 3.420 (3) $\AA$ and a shortest intercentroid separation of 3.6797 (17) $\AA$. The molecular packing is further stabilized by $\mathrm{C}-\mathrm{H} \cdots \mathrm{O}, \mathrm{C}-\mathrm{H} \cdots \mathrm{Cl}$ and $\mathrm{C}-\mathrm{H} \cdots \pi$ interactions between the molecules of the neighbouring stacks (Fig. 3). Details of all these contacts are given in Table 2 .

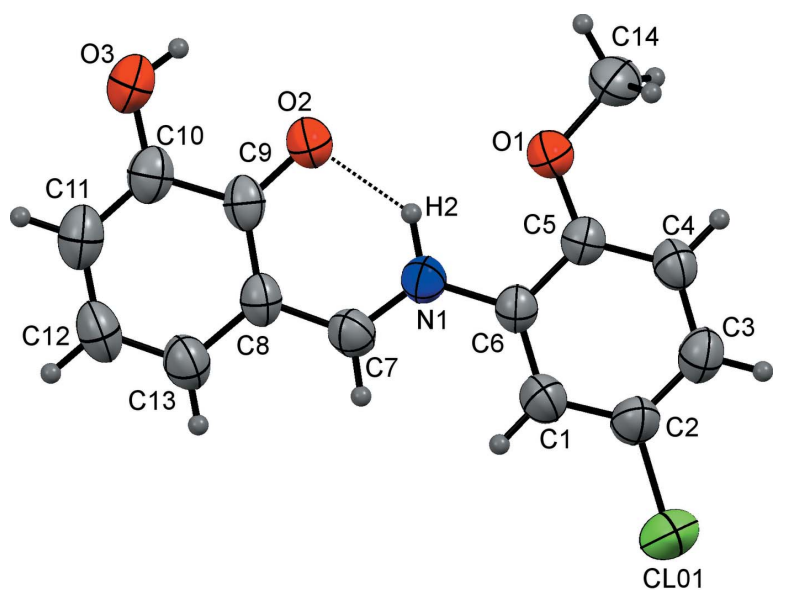

Figure 1

The molecular structure of the title compound with the displacement ellipsoids drawn at the $50 \%$ probability level. The intramolecular $\mathrm{N}-$ $\mathrm{H}$. . O hydrogen bond is shown as a dashed line.

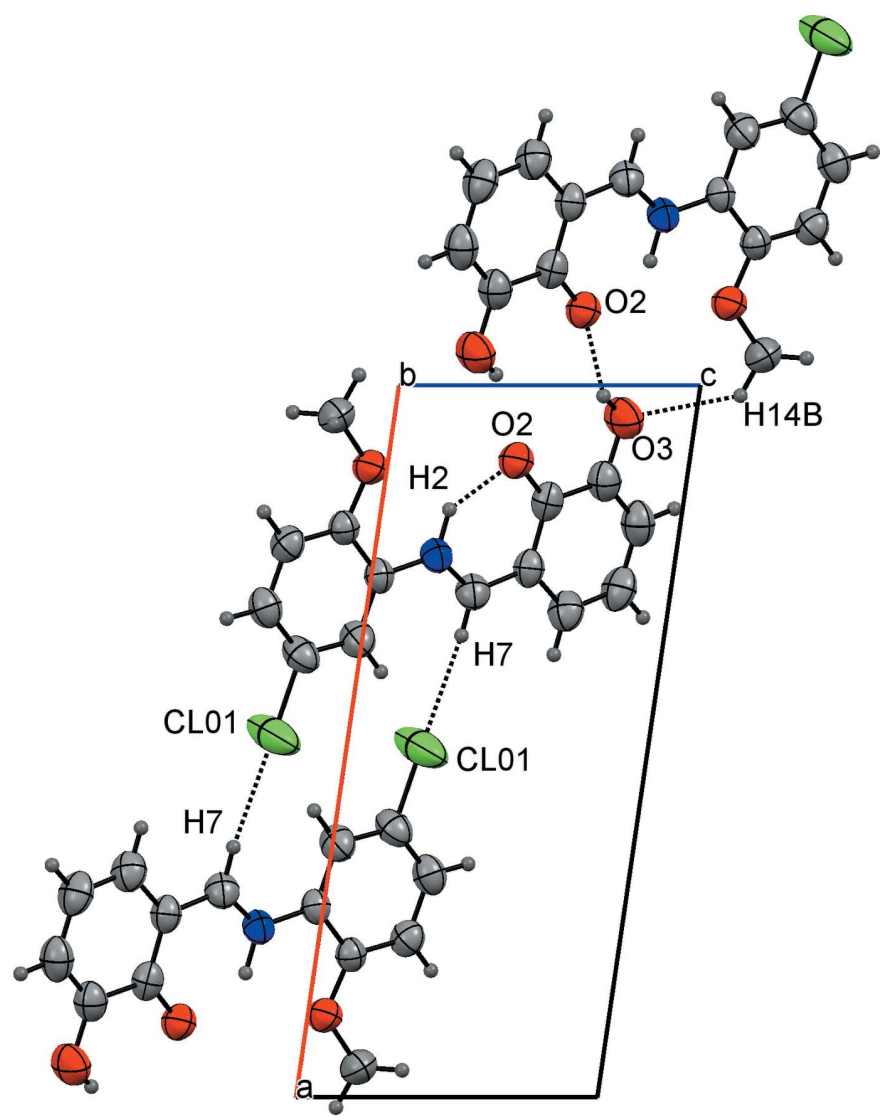

Figure 2

A view of the crystal packing of the title compound. Dashed lines denote the intra- and intermolecular hydrogen bonds.

\section{Database survey}

A search of the Cambridge Structural database (CSD, version 5.40, update November 2018; Groom et al., 2016) for the 3-[(E)-(phenylimino)methyl]-benzene-1,2-diol fragment revealed eight hits where this fragment adopts the ketoenamine tautomeric form and 21 hits where it exists as the phenol-imine tautomer. Distinctive bond lengths (N1-C7,

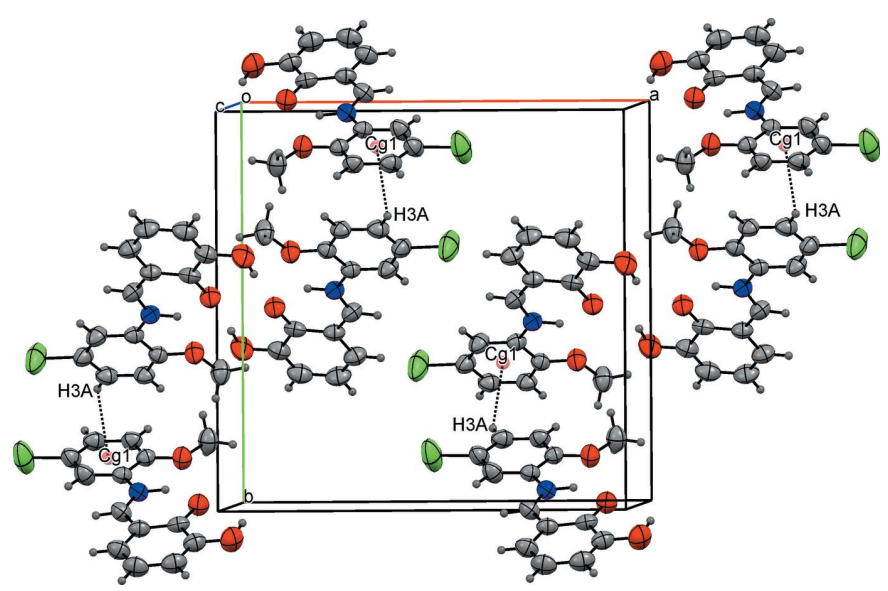

Figure 3

The packing diagram showing the stacking, $\mathrm{C}-\mathrm{H} \cdots \pi$ and $\mathrm{C}-\mathrm{H} \cdots \mathrm{Cl}$ interactions. 
Table 2

Hydrogen-bond geometry $\left(\AA,^{\circ}\right)$.

$C g 1$ is the centroid of the $\mathrm{C} 1-\mathrm{C} 6$ ring.

\begin{tabular}{lllll}
\hline$D-\mathrm{H} \cdots A$ & $D-\mathrm{H}$ & $\mathrm{H} \cdots A$ & $D \cdots A$ & $D-\mathrm{H} \cdots A$ \\
\hline $\mathrm{C} 7-\mathrm{H} 7 \cdots \mathrm{Cl}^{2} 1^{\mathrm{i}}$ & 0.93 & 2.88 & $3.737(3)$ & 154 \\
$\mathrm{C} 14-\mathrm{H} 14 B \cdots \mathrm{O} 3^{\mathrm{ii}}$ & 0.96 & 2.59 & $3.295(4)$ & 131 \\
$\mathrm{O} 3-\mathrm{H} 3 \cdots \mathrm{O} 2^{\mathrm{ii}}$ & $0.87(4)$ & $2.00(4)$ & $2.780(4)$ & $148(4)$ \\
$\mathrm{N} 1-\mathrm{H} 2 \cdots \mathrm{O} 2$ & $0.97(4)$ & $1.82(4)$ & $2.598(3)$ & $136(4)$ \\
$\mathrm{C} 3-\mathrm{H} 3 A \cdots C g 1^{\mathrm{iii}}$ & 0.93 & 2.73 & $3.463(3)$ & 136 \\
\hline
\end{tabular}

Symmetry codes: (i) $-x+1,-y+1,-z$; (ii) $-x,-y+1,-z+1$; (iii) $x,-y+\frac{1}{2}, z-\frac{1}{2}$.

$\mathrm{C} 7=\mathrm{C} 8, \mathrm{C} 8-\mathrm{C} 9, \mathrm{C} 9=\mathrm{O} 2)$ in the title structure are the same within standard uncertainties as the corresponding bond lengths in the structures of 2-hydroxy-6-[(2-methoxyphenyl)aminomethylene]cyclohexa-2,4-dienone (FOCCOQ; Şahin et al., 2005) and 6-[(4-chlorophenylamino)methylene]-2,3-dihydroxycyclohexa-2,4-dien-1-one (CIRTED; Karabiyık et al., 2008). In the structures of typical phenol-imine tautomers, viz., 3-[(3-bromophenyl)iminomethyl]benzene-1,2-diol (CUCZUW; Keleşoğlu et al., 2009b), 3-[(2-bromophenyl)iminomethyl]benzene-1,2-diol (XEYSOK; Temel et al., 2007) and 3-[(4-butylphenyl)iminomethyl]benzene-1,2-diol (XOZJUS; Keleşoğlu et al., 2009a), the C9-O2 and C7-C8 bond lengths are distinctly longer, being in the ranges 1.324$1.355 \AA$ and $1.427-1.447 \AA$, respectively. It is likely that the intermolecular $\mathrm{O}-\mathrm{H}$. . O hydrogen bond, where the keto $\mathrm{O}$ atom acts as an hydrogen-bond acceptor, is an important prerequisite for the tautomeric shift toward the keto-enamine form. In fact, in all eight structures of the keto-enamine tautomers, hydrogen bonds of this type are observed. However, in 16 of 21 structures of phenol-imine tautomers, such hydrogen bonds are also present. This means that there is another unknown reason for the formation of keto-enamine tautomers.

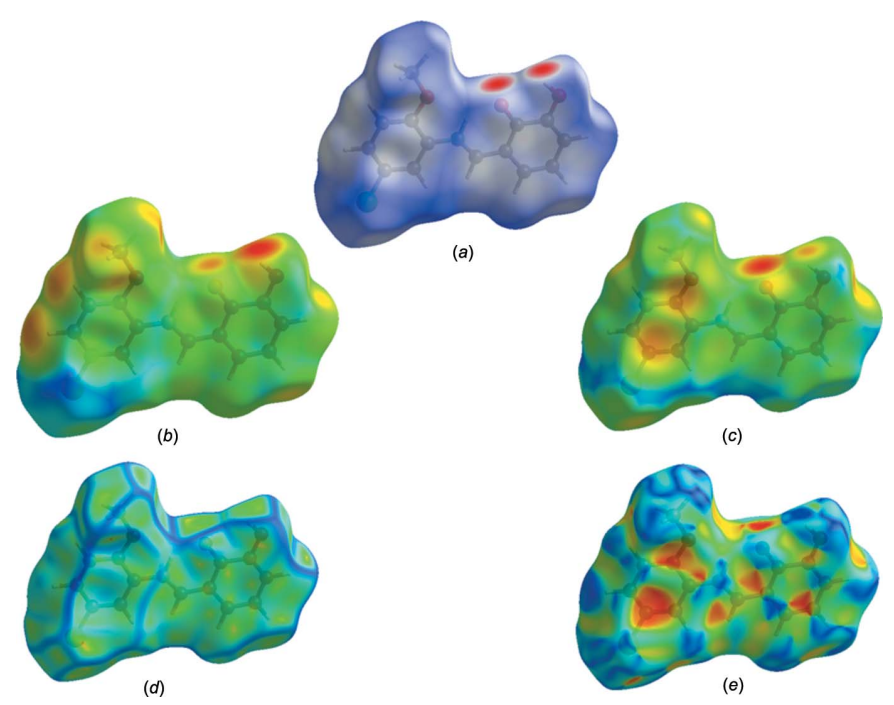

Figure 4

The Hirshfeld surface of the title compound mapped with $(a) d_{\text {norm }},(b) d_{i}$, (c) $d_{e},(d)$ curvedness and (e) shape-index.

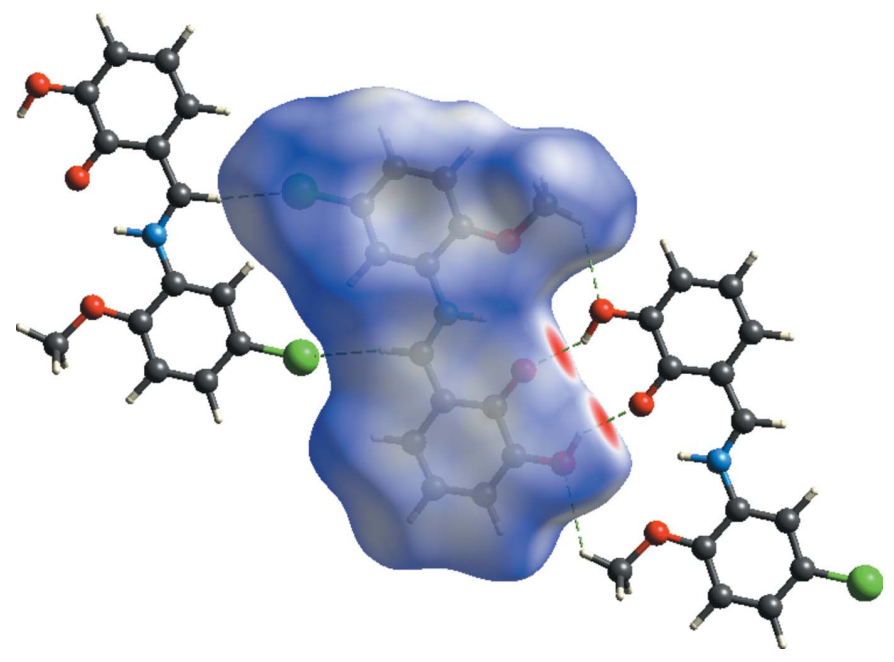

Figure 5

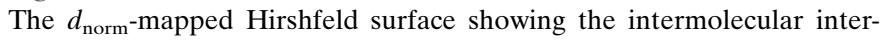
actions in the title compound.

\section{Hirshfeld surface analysis}

The Hirshfeld surface analysis, together with the two-dimensional fingerprint plots, is a powerful tool for the visualization and interpretation of intermolecular contacts in molecular crystals, since it provides a concise description of all intermolecular interactions present in a crystal structure (Spackman \& Jayatilaka, 2009; McKinnon et al., 2007). All surfaces and 2D fingerprint plots were generated using CrystalExplorer3.1 (Wolff et al., 2012). The mappings of $d_{i}, d_{e}$, $d_{\text {norm }}$, shape-index and curvedness for the title structure are shown in Fig. 4. The Hirshfeld surface of a molecule in the crystal is presented in Fig. 5, with the prominent hydrogenbonding interactions shown as intense red spots. The twodimensional fingerprint plots provide information about the

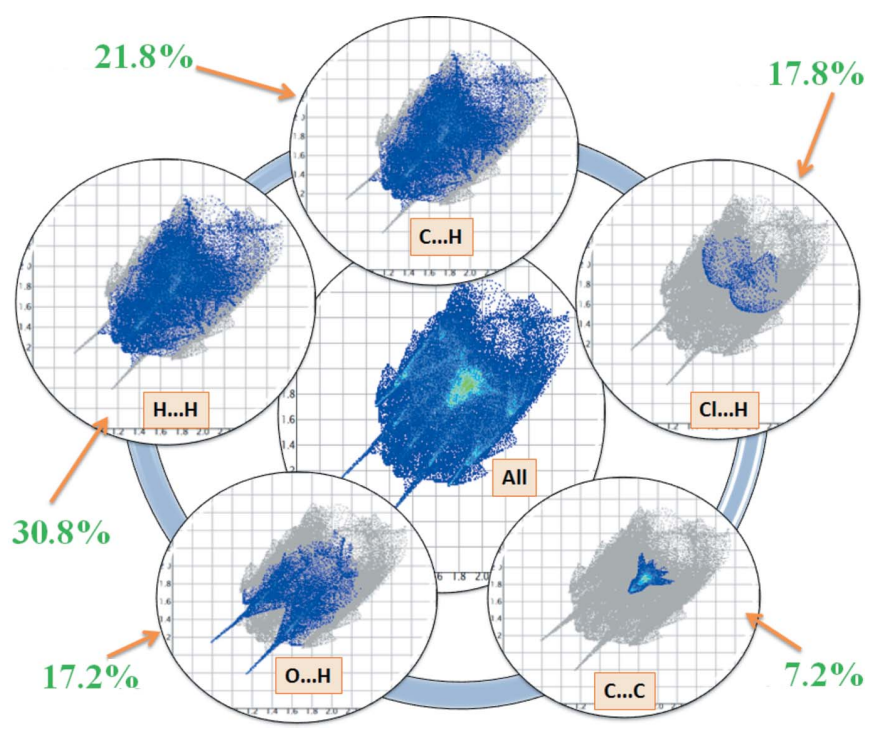

Figure 6

Two-dimensional fingerprint plots with $d_{\text {norm }}$ views of all, the $\mathrm{H} \cdots \mathrm{H}$, $\mathrm{O} \cdots \mathrm{H} / \mathrm{H} \cdots \mathrm{O}, \mathrm{C} \cdots \mathrm{H} / \mathrm{H} \cdots \mathrm{C}$ and $\mathrm{N} \cdots \mathrm{H} / \mathrm{H} \cdots \mathrm{N}$ contacts in the title compound. 


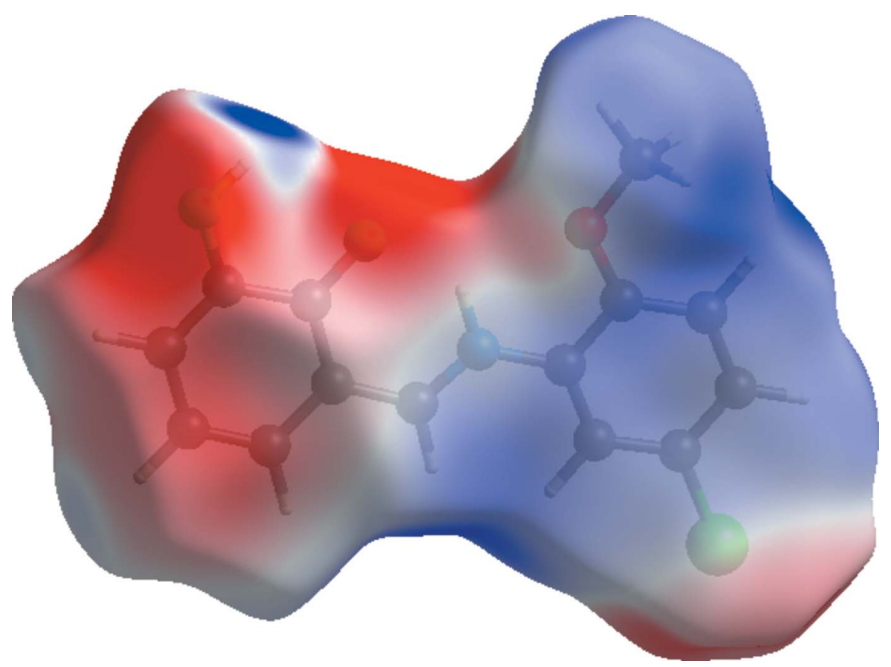

Figure 7

The view of the Hirshfeld surface of the title compound plotted over the electrostatic potential energy.

percentage contributions of the various interatomic contacts. As can be seen from these plots (Fig. 6), the most important are the $\mathrm{H} \cdots \mathrm{H}$ interactions, which contribute $30.8 \%$ to the total Hirshfeld surface. Other contributions are from $\mathrm{O} \cdots \mathrm{C} /$ C. . O (1.2\%), O $\cdots \mathrm{H} / \mathrm{H} \cdots \mathrm{O}(17.2 \%), \mathrm{C} \cdots \mathrm{C}(7.2 \%), \mathrm{O} \cdots \mathrm{O} /$ $\mathrm{O} \cdots \mathrm{O}(1.0 \%), \mathrm{Cl} \cdots \mathrm{H} / \mathrm{H} \cdots \mathrm{Cl}(17.8 \%)$ and $\mathrm{C} \cdot \mathrm{H} / \mathrm{H} \cdots \mathrm{C}$ $(21.8 \%)$. Analogous features were observed recently for some compounds of the same class (Kansiz et al., 2018; Özek Yildirim et al., 2018). The donor and acceptor centers of the hydrogen bonding are represented as blue (positive) and red (negative) regions on the Hirshfeld surface mapped over the electrostatic potential (Fig. 7). The electrostatic potential of the $\mathrm{Cl01}$ atom is less negative as compared to those of atoms $\mathrm{O} 2$ and $\mathrm{O} 3$ of the hydroxy groups, as indicated by the lighter red color.

\section{Synthesis and crystallization}

The title compound was prepared by mixing solutions of 2,3dihydroxybenzaldehyde $(34.5 \mathrm{mg}, 0.25 \mathrm{mmol})$ and 5-chloro-2methoxyaniline $(39.4 \mathrm{mg}, 0.25 \mathrm{mmol})$, both in $15 \mathrm{~mL}$ of ethanol, with subsequent stirring for $5 \mathrm{~h}$ under reflux. Single crystals were obtained by slow evaporation of an ethanol solution (yield 65\%; m.p. 442-444 K).

\section{Refinement}

Crystal data, data collection and structure refinement details are summarized in Table 3. The $\mathrm{C}$-bound $\mathrm{H}$ atoms were geometrically positioned with $\mathrm{C}-\mathrm{H}$ distances of $0.93-0.96 \AA$ and refined as riding, with $U_{\text {iso }}(\mathrm{H})=1.2 U_{\text {eq }}(\mathrm{C})$ or $U_{\text {iso }}(\mathrm{H})=$ $1.5 U_{\text {eq }}(\mathrm{C})$ for methyl $\mathrm{H}$ atoms. The $\mathrm{O}$ - and $\mathrm{N}$-bound $\mathrm{H}$ atoms were located in a difference map and freely refined.

\section{Acknowledgements}

The authors acknowledge the Faculty of Arts and Sciences, Ondokuz Mayıs University, Turkey, for the use of the Stoe
Table 3

Experimental details.

\begin{tabular}{ll}
\hline Crystal data & \\
Chemical formula & $\mathrm{C}_{14} \mathrm{H}_{12} \mathrm{ClNO}_{3}$ \\
$M_{\mathrm{r}}$ & 277.70 \\
Crystal system, space group & Monoclinic, $P 2_{1} / c$ \\
Temperature $(\mathrm{K})$ & 296 \\
$a, b, c(\AA)$ & $14.7251(9), 14.4444(9), 6.1698(4)$ \\
$\beta\left({ }^{\circ}\right)$ & $98.241(5)$ \\
$V\left(\AA^{3}\right)$ & $1298.74(14)$ \\
$Z$ & 4 \\
Radiation type & Mo $K \alpha$ \\
$\mu\left(\mathrm{mm}^{-1}\right)$ & 0.30 \\
Crystal size (mm) & $0.23 \times 0.16 \times 0.09$ \\
& \\
Data collection & Stoe IPDS 2 \\
Diffractometer & Integration $(X-R E D 32 ;$ \\
Absorption correction & Stoe \& Cie, 2002) \\
& $0.948,0.979$ \\
$T_{\text {min }}, T_{\text {max }}$ & $13658,2491,1120$ \\
No. of measured, independent and & \\
$\quad$ observed $[I>2 \sigma(I)]$ reflections & 0.115 \\
$R_{\text {int }}$ & 0.617 \\
$(\text { sin } \theta / \lambda)_{\text {max }}\left(\AA^{-1}\right)$ & \\
Refinement & \\
$R\left[F^{2}>2 \sigma\left(F^{2}\right)\right], w R\left(F^{2}\right), S$ & $0.057,0.100,0.90$ \\
No. of reflections & 2491 \\
No. of parameters & 181 \\
$\mathrm{H}$-atom treatment & $\mathrm{H}$ atoms treated by a mixture of \\
& independent and constrained \\
$\Delta \rho_{\text {max }}, \Delta \rho_{\text {min }}\left(\mathrm{e} \AA^{-3}\right)$ & refinement \\
\hline & $0.16,-0.24$ \\
\hline
\end{tabular}

Computer programs: $X$-AREA and $X$-RED (Stoe \& Cie, 2002), SHELXT (Sheldrick, 2015a), SHELXL2014 (Sheldrick, 2015b), ORTEP-3 for Windows and WinGX (Farrugia, 2012) and PLATON (Spek, 2009).

IPDS 2 diffractometer (purchased under grant F.279 of the University Research Fund).

\section{References}

Calligaris, M. \& Randaccio, L. (1987). Comprehensive Coordination Chemistry, Vol. 2, edited by G. Wilkinson, pp. 715-738. London: Pergamon.

Cohen, M. D., Schmidt, G. M. J. \& Flavian, S. (1964). J. Chem. Soc., 2041-2051.

Costamagna, J., Vargas, J., Latorre, R., Alvarado, A. \& Mena, G. (1992). Coord. Chem. Rev. 119, 67-88.

Farrugia, L. J. (2012). J. Appl. Cryst. 45, 849-854.

Groom, C. R., Bruno, I. J., Lightfoot, M. P. \& Ward, S. C. (2016). Acta Cryst. B72, 171-179.

Hadjoudis, E., Vittorakis, M. \& Moustakali-Mavridis, I. (1987). Tetrahedron, 43, 1345-1360.

Kansız, S., Macit, M., Dege, N. \& Pavlenko, V. A. (2018). Acta Cryst. E74, 1887-1890.

Karabıyık, H., Ocak-İskeleli, N., Petek, H., Albayrak, Ç. \& Ağar, E. (2008). J. Mol. Struct. 873, 130-136.

Keleşoğlu, Z., Büyükgüngör, O., Albayrak, Ç. \& Odabaşoğlu, M. (2009a). Acta Cryst. E65, o2022.

Keleşoğlu, Z., Büyükgüngör, O., Albayrak, Ç. \& Odabaşoğlu, M. (2009b). Acta Cryst. E65, o2410-o2411.

Lozier, R., Bogomolni, R. A. \& Stoeckenius, W. (1975). Biophys. J. 15, 955-962.

McKinnon, J. J., Jayatilaka, D. \& Spackman, M. A. (2007). Chem. Commun. pp. 3814-3816.

Özek Yıldırım, A., Gülsu, M. \& Albayrak Kaştaş, Ç. (2018). Acta Cryst. E74, 319-322. 
Petek, H., Albayrak, Ç., Odabaşoğlu, M., Şenel, I. \& Büyükgüngör, O. (2010). Struct. Chem. 21, 681-690.

Şahin, O., Büyükgüngör, O., Albayrak, C. \& Odabasoglu, M. (2005). Acta Cryst. E61, o1579-o1581.

Sheldrick, G. M. (2015a). Acta Cryst. A71, 3-8.

Sheldrick, G. M. (2015b). Acta Cryst. C71, 3-8.

Spackman, M. A. \& Jayatilaka, D. (2009). CrystEngComm, 11, 19-32.

Spek, A. L. (2009). Acta Cryst. D65, 148-155.
Stewart, J. M. \& Lingafelter, E. C. (1959). Acta Cryst. 12, 842-845.

Stoe \& Cie (2002). $X$-AREA and $X$-RED32. Stoe \& Cie, Darmstadt, Germany.

Temel, E., Albayrak, Ç., Odabaşoğlu, M. \& Büyükgüngör, O. (2007). Acta Cryst. E63, o1319-o1320.

Wolff, S. K., Grimwood, D. J., McKinnon, J. J., Turner, M. J., Jayatilaka, D. \& Spackman, M. A. (2012). CrystalExplorer3.1. University of Western Australia. 


\section{supporting information}

Acta Cryst. (2019). E75, 362-366 [https://doi.org/10.1107/S2056989019002123]

Crystal structure and Hirshfeld surface analysis of a Schiff base: (Z)-6-[(5chloro-2-methoxyanilino)methylidene]-2-hydroxycyclohexa-2,4-dien-1-one

Sibel Demir Kanmazalp, Onur Erman Doĝan, Volkan Taşdemir, Necmi Dege, Erbil Aĝar and Igor O. Fritsky

Computing details

Data collection: $X$-AREA (Stoe \& Cie, 2002); cell refinement: $X$-AREA (Stoe \& Cie, 2002); data reduction: $X$-RED (Stoe \& Cie, 2002); program(s) used to solve structure: SHELXT (Sheldrick, 2015a); program(s) used to refine structure: SHELXL2014 (Sheldrick, 2015b); molecular graphics: ORTEP-3 for Windows (Farrugia, 2012); software used to prepare material for publication: WinGX (Farrugia, 2012)and PLATON (Spek, 2009).

(Z)-6-[(5-Chloro-2-methoxyanilino)methylidene]-2-hydroxycyclohexa-2,4-dien-1-one

Crystal data

$\mathrm{C}_{14} \mathrm{H}_{12} \mathrm{ClNO}_{3}$

$M_{r}=277.70$

Monoclinic, $P 2_{1} / c$

$a=14.7251$ (9) $\AA$

$b=14.4444$ (9) $\AA$

$c=6.1698(4) \AA$

$\beta=98.241(5)^{\circ}$

$V=1298.74(14) \AA^{3}$

$Z=4$

\section{Data collection}

Stoe IPDS 2 diffractometer

Radiation source: sealed X-ray tube, 12 x 0.4 mm long-fine focus

Detector resolution: 6.67 pixels $\mathrm{mm}^{-1}$ rotation method scans

Absorption correction: integration

(X-RED32; Stoe \& Cie, 2002)

$T_{\min }=0.948, T_{\max }=0.979$

Refinement

Refinement on $F^{2}$

Least-squares matrix: full

$R\left[F^{2}>2 \sigma\left(F^{2}\right)\right]=0.057$

$w R\left(F^{2}\right)=0.100$

$S=0.90$

2491 reflections

181 parameters
$F(000)=576$

$D_{\mathrm{x}}=1.420 \mathrm{Mg} \mathrm{m}^{-3}$

Mo $K \alpha$ radiation, $\lambda=0.71073 \AA$

Cell parameters from 8086 reflections

$\theta=1.4-27.1^{\circ}$

$\mu=0.30 \mathrm{~mm}^{-1}$

$T=296 \mathrm{~K}$

Irregular specimen, red

$0.23 \times 0.16 \times 0.09 \mathrm{~mm}$

13658 measured reflections

2491 independent reflections

1120 reflections with $I>2 \sigma(I)$

$R_{\text {int }}=0.115$

$\theta_{\max }=26.0^{\circ}, \theta_{\min }=2.0^{\circ}$

$h=-18 \rightarrow 18$

$k=-17 \rightarrow 17$

$l=-7 \rightarrow 7$

0 restraints

Primary atom site location: structure-invariant direct methods

Secondary atom site location: difference Fourier map

Hydrogen site location: mixed 
$\mathrm{H}$ atoms treated by a mixture of independent and constrained refinement

$w=1 /\left[\sigma^{2}\left(F_{\mathrm{o}}^{2}\right)+(0.0293 P)^{2}\right]$

where $P=\left(F_{\mathrm{o}}^{2}+2 F_{\mathrm{c}}^{2}\right) / 3$

$$
\begin{aligned}
& (\Delta / \sigma)_{\max }<0.001 \\
& \Delta \rho_{\max }=0.16 \mathrm{e} \AA^{-3} \\
& \Delta \rho_{\min }=-0.24 \mathrm{e} \AA^{-3}
\end{aligned}
$$

Special details

Geometry. All esds (except the esd in the dihedral angle between two 1.s. planes) are estimated using the full covariance matrix. The cell esds are taken into account individually in the estimation of esds in distances, angles and torsion angles; correlations between esds in cell parameters are only used when they are defined by crystal symmetry. An approximate

\begin{tabular}{|c|c|c|c|c|}
\hline & $x$ & $y$ & $z$ & $U_{\text {iso }} * / U_{\text {eq }}$ \\
\hline $\mathrm{C} 101$ & $0.49115(8)$ & $0.36774(9)$ & $-0.2476(2)$ & $0.1172(6)$ \\
\hline $\mathrm{O} 1$ & $0.11503(15)$ & $0.37573(16)$ & $-0.0556(4)$ & $0.0611(6)$ \\
\hline $\mathrm{O} 2$ & $0.10563(15)$ & $0.50349(16)$ & $0.4247(4)$ & $0.0601(7)$ \\
\hline $\mathrm{O} 3$ & $0.0461(2)$ & $0.5981(2)$ & $0.7618(4)$ & $0.0782(9)$ \\
\hline N1 & $0.2392(2)$ & $0.46609(18)$ & $0.2056(5)$ & $0.0497(8)$ \\
\hline $\mathrm{C} 1$ & $0.3568(2)$ & $0.4173(2)$ & $-0.0160(6)$ & $0.0608(10)$ \\
\hline H1A & 0.4026 & 0.4475 & 0.0774 & $0.073^{*}$ \\
\hline $\mathrm{C} 2$ & $0.3782(2)$ & $0.3706(2)$ & $-0.1958(6)$ & $0.0606(10)$ \\
\hline $\mathrm{C} 3$ & $0.3124(2)$ & $0.3262(2)$ & $-0.3362(6)$ & $0.0583(10)$ \\
\hline $\mathrm{H} 3 \mathrm{~A}$ & 0.3277 & 0.2961 & -0.4592 & $0.070^{*}$ \\
\hline $\mathrm{C} 4$ & $0.2231(2)$ & $0.3264(2)$ & $-0.2937(6)$ & $0.0540(9)$ \\
\hline H4 & 0.1780 & 0.2953 & -0.3869 & $0.065^{*}$ \\
\hline $\mathrm{C} 5$ & $0.2002(2)$ & $0.3725(2)$ & $-0.1142(5)$ & $0.0459(8)$ \\
\hline $\mathrm{C} 6$ & $0.2675(2)$ & $0.4193(2)$ & $0.0256(5)$ & $0.0454(8)$ \\
\hline $\mathrm{C} 7$ & $0.2902(2)$ & $0.5189(2)$ & $0.3445(6)$ & $0.0546(9)$ \\
\hline H7 & 0.3515 & 0.5266 & 0.3277 & $0.066^{*}$ \\
\hline $\mathrm{C} 8$ & $0.2572(2)$ & $0.5648(2)$ & $0.5188(5)$ & $0.0495(9)$ \\
\hline C9 & $0.1632(2)$ & $0.5551(2)$ & $0.5481(5)$ & $0.0483(9)$ \\
\hline $\mathrm{C} 10$ & $0.1348(3)$ & $0.6045(2)$ & $0.7269(6)$ & $0.0577(9)$ \\
\hline $\mathrm{C} 11$ & $0.1941(3)$ & $0.6591(2)$ & $0.8593(6)$ & $0.0669(11)$ \\
\hline H11 & 0.1733 & 0.6919 & 0.9723 & $0.080^{*}$ \\
\hline C12 & $0.2862(3)$ & $0.6667(2)$ & $0.8274(6)$ & $0.0680(11)$ \\
\hline H12 & 0.3262 & 0.7037 & 0.9205 & $0.082^{*}$ \\
\hline $\mathrm{C} 13$ & $0.3171(3)$ & $0.6206(2)$ & $0.6623(6)$ & $0.0619(10)$ \\
\hline H13 & 0.3784 & 0.6257 & 0.6430 & $0.074^{*}$ \\
\hline $\mathrm{C} 14$ & $0.0424(2)$ & $0.3301(3)$ & $-0.1933(6)$ & $0.0698(11)$ \\
\hline $\mathrm{H} 00 \mathrm{~F}$ & 0.0378 & 0.3546 & -0.3392 & $0.105^{*}$ \\
\hline H14B & -0.0144 & 0.3402 & -0.1375 & $0.105^{*}$ \\
\hline $\mathrm{H} 14 \mathrm{C}$ & 0.0548 & 0.2649 & -0.1959 & $0.105^{*}$ \\
\hline H3 & $0.016(3)$ & 0.555 & $0.685(8)$ & $0.104(18)^{*}$ \\
\hline H2 & $0.175(3)$ & $0.461(3)$ & $0.226(8)$ & $0.130(17)^{*}$ \\
\hline
\end{tabular}
(isotropic) treatment of cell esds is used for estimating esds involving l.s. planes.

Fractional atomic coordinates and isotropic or equivalent isotropic displacement parameters $\left(\AA^{2}\right)$ 
Atomic displacement parameters $\left(\AA^{2}\right)$

\begin{tabular}{lllllll}
\hline & $U^{11}$ & $U^{22}$ & $U^{33}$ & $U^{12}$ & $U^{13}$ & $U^{23}$ \\
\hline C101 & $0.0754(8)$ & $0.1458(11)$ & $0.1426(12)$ & $-0.0326(7)$ & $0.0573(8)$ & $-0.0628(9)$ \\
O1 & $0.0504(14)$ & $0.0746(16)$ & $0.0578(16)$ & $-0.0021(13)$ & $0.0062(12)$ & $-0.0180(13)$ \\
O2 & $0.0581(15)$ & $0.0667(16)$ & $0.0544(17)$ & $-0.0025(13)$ & $0.0051(13)$ & $-0.0148(12)$ \\
O3 & $0.077(2)$ & $0.087(2)$ & $0.074(2)$ & $0.0025(16)$ & $0.0234(17)$ & $-0.0277(16)$ \\
N1 & $0.0535(19)$ & $0.0501(19)$ & $0.047(2)$ & $-0.0040(14)$ & $0.0114(17)$ & $-0.0032(14)$ \\
C1 & $0.063(3)$ & $0.060(2)$ & $0.061(3)$ & $-0.0134(17)$ & $0.014(2)$ & $-0.0135(18)$ \\
C2 & $0.061(2)$ & $0.057(2)$ & $0.068(3)$ & $-0.011(2)$ & $0.025(2)$ & $-0.010(2)$ \\
C3 & $0.073(3)$ & $0.050(2)$ & $0.056(3)$ & $0.001(2)$ & $0.019(2)$ & $-0.0059(19)$ \\
C4 & $0.060(3)$ & $0.048(2)$ & $0.053(2)$ & $-0.0013(17)$ & $0.005(2)$ & $-0.0097(17)$ \\
C5 & $0.051(2)$ & $0.043(2)$ & $0.044(2)$ & $0.0025(17)$ & $0.0085(18)$ & $0.0000(17)$ \\
C6 & $0.055(2)$ & $0.041(2)$ & $0.041(2)$ & $-0.0018(16)$ & $0.0093(19)$ & $-0.0023(15)$ \\
C7 & $0.059(2)$ & $0.052(2)$ & $0.053(2)$ & $-0.0125(18)$ & $0.010(2)$ & $-0.0031(19)$ \\
C8 & $0.063(2)$ & $0.042(2)$ & $0.043(2)$ & $-0.0008(16)$ & $0.0073(19)$ & $-0.0044(16)$ \\
C9 & $0.067(2)$ & $0.0347(19)$ & $0.042(2)$ & $0.0064(17)$ & $0.004(2)$ & $-0.0009(16)$ \\
C10 & $0.072(3)$ & $0.050(2)$ & $0.052(2)$ & $0.007(2)$ & $0.011(2)$ & $-0.0005(19)$ \\
C11 & $0.093(3)$ & $0.056(3)$ & $0.053(3)$ & $0.007(2)$ & $0.015(2)$ & $-0.0073(19)$ \\
C12 & $0.088(3)$ & $0.058(2)$ & $0.055(3)$ & $-0.011(2)$ & $0.000(2)$ & $-0.015(2)$ \\
C13 & $0.074(2)$ & $0.054(2)$ & $0.057(3)$ & $-0.010(2)$ & $0.008(2)$ & $-0.0087(19)$ \\
C14 & $0.053(2)$ & $0.095(3)$ & $0.060(3)$ & $-0.002(2)$ & $0.002(2)$ & $-0.010(2)$ \\
& & & & & & \\
\hline
\end{tabular}

Geometric parameters $\left(A,{ }^{\circ}\right)$

\begin{tabular}{llll}
\hline $\mathrm{C} 101-\mathrm{C} 2$ & $1.739(3)$ & $\mathrm{C} 4-\mathrm{H} 4$ & 0.9300 \\
$\mathrm{O} 1-\mathrm{C} 5$ & $1.354(3)$ & $\mathrm{C} 5-\mathrm{C} 6$ & $1.393(4)$ \\
$\mathrm{O} 1-\mathrm{C} 14$ & $1.429(4)$ & $\mathrm{C} 7-\mathrm{C} 8$ & $1.408(4)$ \\
$\mathrm{O} 2-\mathrm{C} 9$ & $1.292(4)$ & $\mathrm{C} 7-\mathrm{H} 7$ & 0.9300 \\
$\mathrm{O} 3-\mathrm{C} 10$ & $1.358(4)$ & $\mathrm{C} 8-\mathrm{C} 9$ & $1.429(4)$ \\
$\mathrm{O} 3-\mathrm{H} 3$ & $0.87(4)$ & $\mathrm{C} 8-\mathrm{C} 13$ & $1.410(5)$ \\
$\mathrm{N} 1-\mathrm{C} 6$ & $1.413(4)$ & $\mathrm{C} 9-\mathrm{C} 10$ & $1.426(4)$ \\
$\mathrm{N} 1-\mathrm{C} 7$ & $1.302(4)$ & $\mathrm{C} 10-\mathrm{C} 11$ & $1.359(5)$ \\
$\mathrm{N} 1-\mathrm{H} 2$ & $0.97(4)$ & $\mathrm{C} 11-\mathrm{C} 12$ & $1.402(5)$ \\
$\mathrm{C} 1-\mathrm{C} 2$ & $1.372(4)$ & $\mathrm{C} 11-\mathrm{H} 11$ & 0.9300 \\
$\mathrm{C} 1-\mathrm{C} 6$ & $1.376(4)$ & $\mathrm{C} 12-\mathrm{C} 13$ & $1.349(4)$ \\
$\mathrm{C} 1-\mathrm{H} 1 \mathrm{~A}$ & 0.9300 & $\mathrm{C} 12-\mathrm{H} 12$ & 0.9300 \\
$\mathrm{C} 2-\mathrm{C} 3$ & $1.363(5)$ & $\mathrm{C} 13-\mathrm{H} 13$ & 0.9300 \\
$\mathrm{C} 3-\mathrm{C} 4$ & $1.379(4)$ & $\mathrm{C} 14-\mathrm{H} 00 \mathrm{~F}$ & 0.9600 \\
$\mathrm{C} 3-\mathrm{H} 3 \mathrm{~A}$ & 0.9300 & $\mathrm{C} 14-\mathrm{H} 14 \mathrm{~B}$ & 0.9600 \\
$\mathrm{C} 4-\mathrm{C} 5$ & $1.375(4)$ & $\mathrm{C} 14-\mathrm{H} 14 \mathrm{C}$ & 0.9600 \\
& & & 118.3 \\
$\mathrm{C} 5-\mathrm{O} 1-\mathrm{C} 14$ & $117.9(3)$ & $\mathrm{C} 8-\mathrm{C} 7-\mathrm{H} 7$ & $119.7(3)$ \\
$\mathrm{C} 10-\mathrm{O} 3-\mathrm{H} 3$ & $113(3)$ & $\mathrm{C} 7-\mathrm{C} 8-\mathrm{C} 13$ & $119.8(3)$ \\
$\mathrm{C} 7-\mathrm{N} 1-\mathrm{C} 6$ & $126.1(3)$ & $\mathrm{C} 7-\mathrm{C} 8-\mathrm{C} 9$ & $120.4(3)$ \\
$\mathrm{C} 7-\mathrm{N} 1-\mathrm{H} 2$ & $116(3)$ & $\mathrm{C} 13-\mathrm{C} 8-\mathrm{C} 9$ & $120.3(3)$ \\
$\mathrm{C} 6-\mathrm{N} 1-\mathrm{H} 2$ & $118(3)$ & $\mathrm{O} 2-\mathrm{C} 9-\mathrm{C} 10$ & $123.2(3)$ \\
$\mathrm{C} 2-\mathrm{C} 1-\mathrm{C} 6$ & $119.8(3)$ & $\mathrm{O} 2-\mathrm{C} 9-\mathrm{C} 8$ &
\end{tabular}




$\begin{array}{ll}\mathrm{C} 2-\mathrm{C} 1-\mathrm{H} 1 \mathrm{~A} & 120.1 \\ \mathrm{C} 6-\mathrm{C} 1-\mathrm{H} 1 \mathrm{~A} & 120.1 \\ \mathrm{C} 3-\mathrm{C} 2-\mathrm{C} 1 & 121.4(3) \\ \mathrm{C} 3-\mathrm{C} 2-\mathrm{C} 101 & 118.9(3) \\ \mathrm{C} 1-\mathrm{C} 2-\mathrm{C} 101 & 119.7(3) \\ \mathrm{C} 2-\mathrm{C} 3-\mathrm{C} 4 & 119.2(3) \\ \mathrm{C} 2-\mathrm{C} 3-\mathrm{H} 3 \mathrm{~A} & 120.4 \\ \mathrm{C} 4-\mathrm{C} 3-\mathrm{H} 3 \mathrm{~A} & 120.4 \\ \mathrm{C} 5-\mathrm{C} 4-\mathrm{C} 3 & 120.4(3) \\ \mathrm{C} 5-\mathrm{C} 4-\mathrm{H} 4 & 119.8 \\ \mathrm{C} 3-\mathrm{C} 4-\mathrm{H} 4 & 119.8 \\ \mathrm{O} 1-\mathrm{C} 5-\mathrm{C} 4 & 125.1(3) \\ \mathrm{O} 1-\mathrm{C} 5-\mathrm{C} 6 & 115.0(3) \\ \mathrm{C} 4-\mathrm{C} 5-\mathrm{C} 6 & 119.9(3) \\ \mathrm{C} 1-\mathrm{C} 6-\mathrm{C} 5 & 119.3(3) \\ \mathrm{C} 1-\mathrm{C} 6-\mathrm{N} 1 & 123.7(3) \\ \mathrm{C} 5-\mathrm{C} 6-\mathrm{N} 1 & 117.0(3) \\ \mathrm{N} 1-\mathrm{C} 7-\mathrm{C} 8 & 123.3(3) \\ \mathrm{N} 1-\mathrm{C} 7-\mathrm{H} 7 & 118.3 \\ & \\ \mathrm{C} 6-\mathrm{C} 1-\mathrm{C} 2-\mathrm{C} 3 & 0.3(6) \\ \mathrm{C} 6-\mathrm{C} 1-\mathrm{C} 2-\mathrm{C} 101 & -179.7(3) \\ \mathrm{C} 1-\mathrm{C} 2-\mathrm{C} 3-\mathrm{C} 4 & -1.5(6) \\ \mathrm{C} 101-\mathrm{C} 2-\mathrm{C} 3-\mathrm{C} 4 & 178.6(3) \\ \mathrm{C} 2-\mathrm{C} 3-\mathrm{C} 4-\mathrm{C} 5 & 1.3(5) \\ \mathrm{C} 14-\mathrm{O} 1-\mathrm{C} 5-\mathrm{C} 4 & -1.8(5) \\ \mathrm{C} 14-\mathrm{O} 1-\mathrm{C} 5-\mathrm{C} 6 & 179.2(3) \\ \mathrm{C} 3-\mathrm{C} 4-\mathrm{C} 5-\mathrm{O} 1 & -178.8(3) \\ \mathrm{C} 3-\mathrm{C} 4-\mathrm{C} 5-\mathrm{C} 6 & 0.1(5) \\ \mathrm{C} 2-\mathrm{C} 1-\mathrm{C} 6-\mathrm{C} 5 & 1.0(5) \\ \mathrm{C} 2-\mathrm{C} 1-\mathrm{C} 6-\mathrm{N} 1 & -179.5(3) \\ \mathrm{O} 1-\mathrm{C} 5-\mathrm{C} 6-\mathrm{C} 1 & 177.8(3) \\ \mathrm{C} 4-\mathrm{C} 5-\mathrm{C} 6-\mathrm{C} 1 & -1.2(5) \\ \mathrm{O} 1-\mathrm{C} 5-\mathrm{C} 6-\mathrm{N} 1 & -1.7(4) \\ \mathrm{C} 4-\mathrm{C} 5-\mathrm{C} 6-\mathrm{N} 1 & 179.3(3) \\ \mathrm{C} 7-\mathrm{N} 1-\mathrm{C} 6-\mathrm{C} 1 & 5.8(5) \\ \mathrm{C} 7-\mathrm{N} 1-\mathrm{C} 6-\mathrm{C} 5 & -174.7(3) \\ & \end{array}$

$\mathrm{C} 10-\mathrm{C} 9-\mathrm{C} 8$

$\mathrm{O} 3-\mathrm{C} 10-\mathrm{C} 11$

$\mathrm{O} 3-\mathrm{C} 10-\mathrm{C} 9$

$\mathrm{C} 11-\mathrm{C} 10-\mathrm{C} 9$

$\mathrm{C} 10-\mathrm{C} 11-\mathrm{C} 12$

$\mathrm{C} 10-\mathrm{C} 11-\mathrm{H} 11$

C12-C11-H11

$\mathrm{C} 13-\mathrm{C} 12-\mathrm{C} 11$

$\mathrm{C} 13-\mathrm{C} 12-\mathrm{H} 12$

$\mathrm{C} 11-\mathrm{C} 12-\mathrm{H} 12$

$\mathrm{C} 12-\mathrm{C} 13-\mathrm{C} 8$

$\mathrm{C} 12-\mathrm{C} 13-\mathrm{H} 13$

$\mathrm{C} 8-\mathrm{C} 13-\mathrm{H} 13$

$\mathrm{O} 1-\mathrm{C} 14-\mathrm{H} 00 \mathrm{~F}$

$\mathrm{O} 1-\mathrm{C} 14-\mathrm{H} 14 \mathrm{~B}$

$\mathrm{H} 00 \mathrm{~F}-\mathrm{C} 14-\mathrm{H} 14 \mathrm{~B}$

$\mathrm{O} 1-\mathrm{C} 14-\mathrm{H} 14 \mathrm{C}$

$\mathrm{H} 00 \mathrm{~F}-\mathrm{C} 14-\mathrm{H} 14 \mathrm{C}$

H14B-C14-H14C

$\mathrm{C} 6-\mathrm{N} 1-\mathrm{C} 7-\mathrm{C} 8$

$\mathrm{N} 1-\mathrm{C} 7-\mathrm{C} 8-\mathrm{C} 13$

$\mathrm{N} 1-\mathrm{C} 7-\mathrm{C} 8-\mathrm{C} 9$

$\mathrm{C} 7-\mathrm{C} 8-\mathrm{C} 9-\mathrm{O} 2$

$\mathrm{C} 13-\mathrm{C} 8-\mathrm{C} 9-\mathrm{O} 2$

$\mathrm{C} 7-\mathrm{C} 8-\mathrm{C} 9-\mathrm{C} 10$

$\mathrm{C} 13-\mathrm{C} 8-\mathrm{C} 9-\mathrm{C} 10$

$\mathrm{O} 2-\mathrm{C} 9-\mathrm{C} 10-\mathrm{O} 3$

$\mathrm{C} 8-\mathrm{C} 9-\mathrm{C} 10-\mathrm{O} 3$

$\mathrm{O} 2-\mathrm{C} 9-\mathrm{C} 10-\mathrm{C} 11$

$\mathrm{C} 8-\mathrm{C} 9-\mathrm{C} 10-\mathrm{C} 11$

$\mathrm{O} 3-\mathrm{C} 10-\mathrm{C} 11-\mathrm{C} 12$

$\mathrm{C} 9-\mathrm{C} 10-\mathrm{C} 11-\mathrm{C} 12$

$\mathrm{C} 10-\mathrm{C} 11-\mathrm{C} 12-\mathrm{C} 13$

$\mathrm{C} 11-\mathrm{C} 12-\mathrm{C} 13-\mathrm{C} 8$

$\mathrm{C} 7-\mathrm{C} 8-\mathrm{C} 13-\mathrm{C} 12$

$\mathrm{C} 9-\mathrm{C} 8-\mathrm{C} 13-\mathrm{C} 12$
$116.5(3)$

$119.6(3)$

$118.9(4)$

$121.4(3)$

$120.8(3)$

119.6

119.6

120.3 (4)

119.8

119.8

$120.5(3)$

119.7

119.7

109.5

109.5

109.5

109.5

109.5

109.5

$179.0(3)$

179.9 (3)

$-0.6(5)$

$2.0(5)$

-178.5 (3)

$-179.3(3)$

0.1 (5)

$-1.5(5)$

179.8 (3)

179.8 (3)

$1.2(5)$

$179.7(3)$

-1.7 (6)

0.9 (6)

0.4 (6)

$178.6(3)$

-0.9 (5)

Hydrogen-bond geometry $\left(A,{ }^{\circ}\right)$

$\mathrm{Cg} 1$ is the centroid of the $\mathrm{C} 1-\mathrm{C} 6$ ring.

\begin{tabular}{lllll}
\hline$D-\mathrm{H} \cdots A$ & $D-\mathrm{H}$ & $\mathrm{H} \cdots A$ & $D \cdots A$ & $D-\mathrm{H} \cdots A$ \\
\hline $\mathrm{C} 7-\mathrm{H} 7 \cdots \mathrm{Cl}^{2} 1^{\mathrm{i}}$ & 0.93 & 2.88 & $3.737(3)$ & 154 \\
$\mathrm{C} 14-\mathrm{H} 14 B \cdots \mathrm{O} 3^{\mathrm{ii}}$ & 0.96 & 2.59 & $3.295(4)$ & 131 \\
$\mathrm{O} 3-\mathrm{H} 3 \cdots \mathrm{O} 2^{\mathrm{ii}}$ & $0.87(4)$ & $2.00(4)$ & $2.780(4)$ & $148(4)$
\end{tabular}


$\mathrm{N} 1-\mathrm{H} 2 \cdots \mathrm{O} 2$

$\mathrm{C} 3-\mathrm{H} 3 A \cdots C g 1^{\mathrm{iii}}$

Symmetry codes: (i) $-x+1,-y+1,-z$; (ii) $-x,-y+1,-z+1$; (iii) $x,-y+1 / 2, z-1 / 2$.
1.82 (4)

2.73
$2.598(3)$

$3.463(3)$
$136(4)$

136 